\title{
ISOLATED COR TRIATRIATUM SINISTER: A CASE REPORT
}

\author{
Zanda Grīnberga*, Pauls Sīlis*,**, Elīna Ligere*,**, Ingūna Lubaua*,**, Inta Bergmane**, Luīze Auziņa*,**, Lauris \\ Šmits**, Valts Ozoliň̌̌**, Normunds Sikora*,**, Inga Lāce*,** \\ * Riga Stradins University \\ ** University Hospital for Children, Riga, Latvia Department for Pediatric Cardiology and Cardiac Surgery
}

\begin{abstract}
Summary
Cor triatriatum sinister is a rare congenital cardiac anomaly that has been identified in $0.1 \%$ of children with congenital heart disease. It is defined as a fibromuscular membrane that divides the left atrium into two chambers: a superior (proximal) that in most cases receives drainage from the pulmonary veins and an inferior (distal) chamber that communicates with the mitral valve and the left atrium. Cor triatriatum sinister can be an isolated lesion (approximately 25\% of cases), but in many cases it is associated with other congenital cardiovascular anomalies, the most common one being - atrial septal defect(3). Symptoms in patients with cor triatriatum sinister are related to obstruction of pulmonary venous drainage, pressure loading of the right side of the heart and congestive cardiac failure. Depending on the severity of the obstruction and presence of associated cardiac anomalies it can be diagnosed at any age. Diagnosis is usually achieved by echocardiography in early infancy. Elective treatment method is surgical excision of the membrane. Here we present a pediatric patient (4 months old) presenting in cardiogenic shock with a successful correction of isolated cor triatriatum sinister. To confirm diagnosis and success of surgical repair, transthoracic and transesophageal echocardiography were used.
\end{abstract}

Keywords: Heart defects, congenital heart disease; Cor triatriatum sinister; surgical repair, transthoracic echocardiography.

\section{AIM OF THE DEMONSTRATION}

The aim of this demonstration is to show the management of patient with rare congenital cardiac anomaly - cor triatriatum sinister presenting in cardiogenic shock.

\section{CASE REPORT}

The patient was a 4 months old boy who was transferred to the Children's Clinical University hospital due to acute cardiac insufficiency. The boy was born from a third pregnancy at 36 weeks of gestation. Birth weight was $3232 \mathrm{~g}$ and height $58 \mathrm{~cm}$. The child received exclusive breastfeeding and early infancy period was event free. In the emergency department, the mother complained about the child's general condition, which had worsened over the past 24 hours. The child's skin had become paler, he had a poor appetite, and frequent episodes of apnoe and grunting. After hospitalization, the child's condition was evaluated as critical - unconsciousness, Glasgow Coma scale 5. Poor hemodynamic parameters, discoagulation, chronic hepatic congestion, congestion in pulmonary circuit, retrograde congestion in systemic circuit, poor cardiac output, low peripheral perfusion, poor central circulation, atrial undulation conduction $4: 1$ and $2: 1$, roentgenogram of the chest showed pulmonary edema. The patient received two catecholamine and synchronized cardioversion three times, there was no effect after either. Transthoracic echocardiography demonstrated situs solitus, normal systemic venous return. Severe dilatation of the right sides of the heart. Right ventricle trabecular and stretched. Compressed left ventricle. Above the mitral valve additional membrane was visualized, it had a small opening $\sim 4 \mathrm{~mm}$, a gradient of stenosis was $40 \mathrm{mmHg}$, average $23 \mathrm{mmHg}$. Flow of the abdominal aorta was normal. Ductus arteriosus was closed. The atrial septum was intact.

The severity of the condition was determined by visualized supramitral membrane, which caused retrograde congestion in pulmonal circuit and didn't allow blood to enter the left ventricle (Fig.1). Dilatation of the hearts right side and severely diminished left ventricle volume caused acute cardiovascular failure with serious heart rhythm disorders. Immediate correction of heart disease under cardio-pulmonary bypass was indicated.

During the preoperative period artificial lung ventilation was started, patient received inotropic support - Adrenaline and temporarily Milrinon. Clinical presentation and laboratory findings indicated severe multiorgan damage - Ph 7.133, lactate level $3.23 \mathrm{mmol} / \mathrm{L}$ in capillary blood.

Preoperative diagnosis: congenital heart disease, cor triatriatum sinister, mitral valve stenosis. Heart rhythm disorders - atrial undulation. Heart insufficiency class IV (NYHA).

Surgical repair: Resection of the mitral supravalvular membrane (cor triatriatum sinister correction).

The surgery was performed under general anesthesia, endotracheal intubation. Right before opening the chest, the patient's condition deteriorated with sudden bradycardia, decrease in blood oxygen saturation. Indirect cardiac massage was performed and adrenaline injected. The patient's condition was 
stabilized. The median sternotomy was performed and cardiopulmonary bypass (CPB) was initiated. There was marked fluid accumulation in all cavities - the pericardium, both pleura, and all tissues observed. A moderately hypothermic $\left(31.6^{\circ} \mathrm{C}\right.$ R) CPB was established, aortic occlusion was made and anterograde cardioplegia was used. After cardioplegia and cardiac arrest, right atriotomy was performed. Fossa ovalis in atrial septum was almost closed, remained only a small communication $\sim 2 \mathrm{~mm}$. Through it, the atrial septum was opened and surgeon accessed left atrium under the supramitral membrane. The mitral valve was visualizedit was unchanged. The supramitral membrane was circular in the left atrium, above the mitral valve. The central part of the membrane was perforated, walls separated and membrane removed. Pulmonary veins were inspected, they were draining to the left atrium above the mitral valve and flow was undisturbed. Atrial septal plastic with xenopericardium patch was conducted. To get a better flow from the hearts left side, atrial septum was extended $\sim 10 \mathrm{~mm}$ vertically and sideways. The crossclamping of aorta was finished and was followed by evacuation of air from the heart cavities and aorta. A spontaneous sinus rhythm was established. The remainder of the operation was completed in the usual fashion.

Transesophageal echocardiography showed free flow from pulmonary veins in the left atrium and to mitral valve. Supramitral membrane elements were no longer observed and there was no transmitral gradient. Both ventricles were aligned - symmetrical and septum intact (Fig. 2).

After transferring from the operation room, the patient's initial condition remained serious with laboratory changes in the end organ damage markers (MODS). The condition gradually stabilized and overall the postoperative course was uneventful. No heart rhythm disorders were observed during the postoperative period. The patient was discharged home on the 12 th day post surgery.

\section{DISCUSSION}

Cor triatriatum sinister is a rare congenital cardiac anomaly where the left atrial chamber is anatomically divided with fibromuscular membrane. It was first described by Church in 1868, as a left atrium divided by an abnormal septum(4). The name "cor triatriatum" was given by Borst in 1905 (5).

The embryologic basis of this condition is unclear and controversial (7). Different theories have been attributed to this anomaly. It is thought that the anomalous membrane may be the result of abnormal growth of the septum primum (7). Four different types of morphologic classification have been introduced, but the most common is by Lam et al, which was proposed in 1962 . In $52 \%$ of cases, there is a presence of atrial septal defect between right atrium and proximal chamber, but in $36 \%$ proximal chamber receives all the pulmonary veins and there is notrial septal defect (6) as this was in our case report.
Clinical presentation is variable and depends on the patient's hemodynamic condition which is determined by obstruction and associated cardiac anomalies. After The Mayo Clinic 50-Year Experience research, the most commonly associated anomalies were atrial septal defect $(28 \%)$, patent foramen ovale $(24 \%)$ and tricuspid regurgitation (24\%) (6). The main presenting symptoms for infants are tachypnea, failure to thrive, poor feeding, shock, and cyanosis $(6 ; 8)$.

Three-dimensional transthoracic and transesophageal echocardiographies have been used to better describe the anatomy of this condition, including evaluation of turbulent flow on color Doppler and determination of any gradient across the membrane. Clinical diagnosis is confirmed using transthoracic and transesophageal echocardiograpy $(7 ; 8)$.

The first choice is surgical treatment. Surgical repair is always required in the presence of an obstructive membrane. CPB and cardioplegic arrest are used. A right atriotomy usually allows access to the left atrial membrane which is completely excised taking care not to injure the mitral valve (1).About $60 \%$ of patients have an uncomplicated recovery after surgery. The main complications are supraventricular tachycardia, peritoneal dialysis and seizures. Long-term results are benign $(2 ; 6 ; 8)$.

In summary, cor triatriatum sinister rarely is the cause of cardiogenic shock in newborns. Although the most common causes of cardiogenic shock in newborns are hypoplastic left heart syndrome, aortic coarctation, and others, one should always keep in mind other congenital heart diseases. Cor triatriatum sinister is not a ductus arteriosus dependent heart disease, therefore, patient's preparation prior to surgery with Prostaglandin E is not possible and emergency surgery is the only treatment option. In uncomplicated cases with timely surgical correction, the long-term prognosis and quality of life are excellent.

\section{Conflict of interest: None}




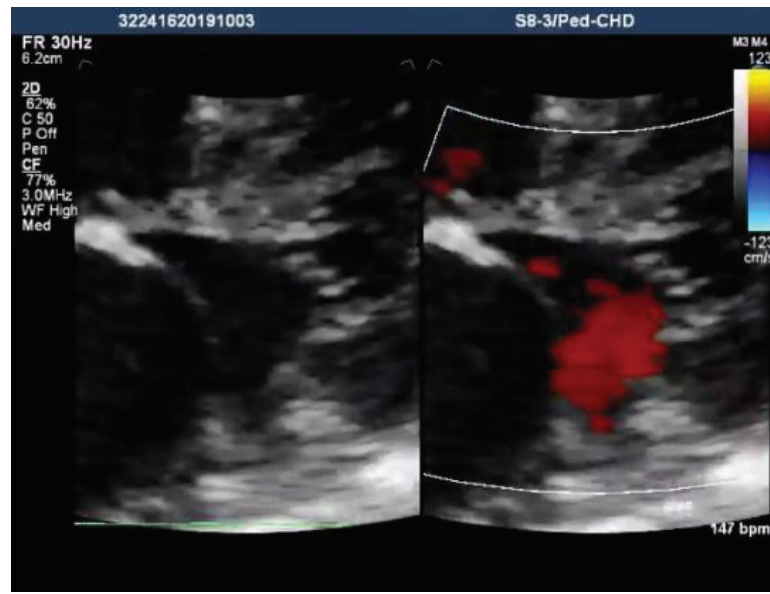

Fig. 1. Transthoracic echocardiography visualized supramitral membrane

\section{REFERENCES}

1. Murthy R, Moe TG, Van Arsdell GS, Nigro JJ, Karamlou T. Congenital Heart Disease // In: Brunicardi F, Andersen DK, Billiar TR, Dunn DL, Kao LS, Hunter JG, Matthews JB, Pollock RE. eds. Schwartz's Principles of Surgery, l le New York, NY: McGraw-Hill; http:// accessmedicine.mhmedical.com.db.rsu.lv/content. aspx? bookid=2576\&sectionid=216221620 (Accessed October 25, 2019)

2. Yaroglu Kazanci, S., Emani, S. and McElhinney, D. Outcome After Repair of Cor Triatriatum // The American Journal of Cardiology, 2012; 109 (3): 412-416

3. Lācis A., Zīdere V., Lubaua I., Straume Z., Auziņš J., Šmits L., Lāce I. Cor Triatrium associated with complex heart defects // Acta Medica Lituanica. 2004; 11: 59-63

4. Church WS. Congenital malformation of the heart: Abnormal septum in left auricle // Trans Pathol Soc London 1867/1868; 19: 188.

5. Borst H. Ein cor triatriatum // Zentralbe Allg Pathol 1905; 16: 812 .

6. Saxena, P., Burkhart, H., Schaff, H., Daly, R., Joyce, L. and Dearani, J. Surgical Repair of Cor Triatriatum Sinister: The Mayo Clinic 50-Year Experience // The Annals of Thoracic Surgery 2014; 97 (5): 1659-1663

7. Thakrar, A., Shapiro, M., Jassal, D., Neilan, T., King, M. and Abbara, S. Cor triatriatum: The utility of cardiovascular imaging // The Canadian journal of Cardiology 2007; 23 (2): 143-145

8. Alphonso, N., Nørgaard, M., Newcomb, A., d'Udekem, Y., Brizard, C. and Cochrane, A. Cor Triatriatum: Presentation, Diagnosis and LongTerm Surgical Results // The Annals of Thoracic Surgery 2005; 80 (5): 1666-1671

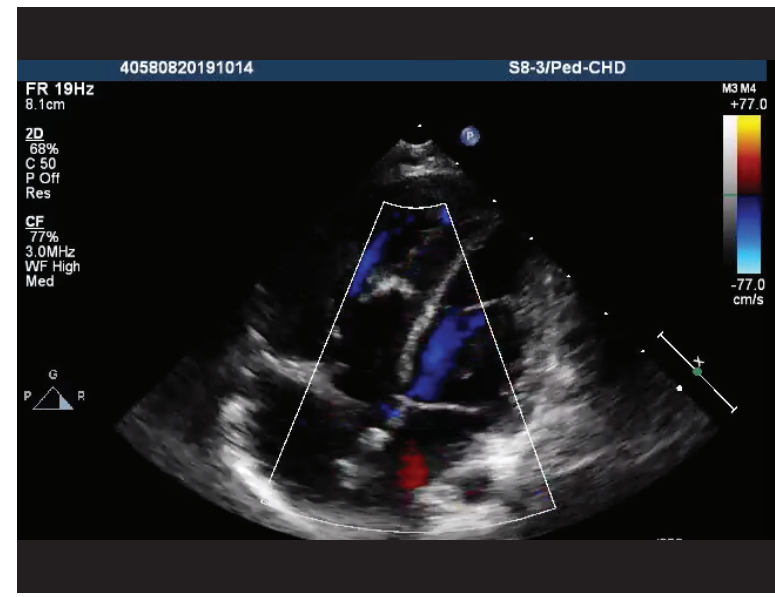

Fig. 2. Post surgery in transthoracic echocardiography supramitral membrane elements were no longer observed, both ventricle were aligned - symmetrical, septum intact

\section{Address:}

Inga Lāce, Zanda Grīnberga

Children's University Hospital,

Department for Paediatric Cardiology and Cardiac Surgery,

Vienibas gatve 45, LV-1004 Riga, Latvia

E-mail:dr.inga.lace@gmail.com,grizanda@inbox.lv 